\title{
Triacylglycerol and Fatty Acid Compositions of Blackberry, Red Raspberry, Black Raspberry, Blueberry and Cranberry Seed Oils by Ultra-Performance Convergence Chromatography- Quadrupole Time-of-Flight Mass Spectrometry
}

\author{
Yinghua Luo ${ }^{1}$, Fanghao Yuan ${ }^{2}$, Yanfang Li ${ }^{2}\left(\mathbb{D}\right.$, Junyi (Danny) Wang ${ }^{3}$, Boyan Gao ${ }^{2,4}, * \mathbb{C}$ and Liangli (Lucy) $\mathrm{Yu}^{4}(\mathbb{D})$ \\ 1 College of Food Science and Nutritional Engineering, National Engineering Research Center for Fruit and \\ Vegetable Processing, Key Laboratory of Fruit and Vegetable Processing Ministry of Agriculture, \\ Engineering Research Centre for Fruit and Vegetable Processing, Ministry of Education, \\ China Agricultural University, Beijing 100083, China; luoyinghua@cau.edu.cn \\ 2 Institute of Food and Nutraceutical Science, School of Agriculture \& Biology, Shanghai Jiao Tong University, \\ Shanghai 200240, China; yuanfanghao@sjtu.edu.cn (F.Y.); zoe_li@sjtu.edu.cn (Y.L.) \\ 3 Bluewood Associaes Co., Ltd., Suzhou 215134, China; danny.wang@bwdasso.com \\ 4 Department of Nutrition and Food Science, University of Maryland, College Park, MD 20742, USA; \\ lyu5@umd.edu \\ * Correspondence: gaoboyan@sjtu.edu.cn; Tel.: +86-3420-4538
}

Citation: Luo, Y.; Yuan, F.; Li, Y.; Wang, J.; Gao, B.; Yu, L. Triacylglycerol and Fatty Acid Compositions of Blackberry, Red Raspberry, Black Raspberry,

Blueberry and Cranberry Seed Oils by Ultra-Performance Convergence Chromatography-Quadrupole Time-of-Flight Mass Spectrometry. Foods 2021, 10, 2530. https:// doi.org/10.3390/foods10112530

Academic Editor: Evaristo Ballesteros

Received: 19 September 2021

Accepted: 19 October 2021

Published: 21 October 2021

Publisher's Note: MDPI stays neutral with regard to jurisdictional claims in published maps and institutional affiliations.

Copyright: (c) 2021 by the authors. Licensee MDPI, Basel, Switzerland. This article is an open access article distributed under the terms and conditions of the Creative Commons Attribution (CC BY) license (https:// creativecommons.org/licenses/by/ $4.0 /)$.

\begin{abstract}
The triacylglycerol (TAG) compositions of blackberry, red raspberry, black raspberry, blueberry and cranberry seed oils were examined using ultra-performance convergence chromatographyquadrupole time-of-flight mass spectrometry (UPC ${ }^{2}$-QTOF MS). A total of 52, 53, 52, 59 and 58 TAGs were detected and tentatively identified from the blackberry, red raspberry, black raspberry, blueberry and cranberry seed oils, respectively, according to their accurate molecular weight in MS1 and fragment ion profiles in MS2. OLL was the most abundant TAG in the blackberry, red raspberry and black raspberry seed oils. Furthermore, the fatty acid compositions of the five berry seed oils were directly determined by gas chromatography coupled with mass spectrometry (GC-MS). In addition, the seed oils had total phenolic contents ranging 13.68-177.06 $\mu$ mol GAE (gallic acid equivalent)/L oil, and significant scavenging capacities against $\mathrm{DPPH}$, peroxyl, and $\mathrm{ABTS}^{+}$radicals. These results indicated that the combination of UPC ${ }^{2}$ and QTOF MS could effectively identify and semi-quantify the TAGs compositions of the berry seed oils with sn-position information for the fatty acids. Understanding the TAGs compositions of these berry seed oils could improve the utilization of these potentially high nutritional value oils for human health.
\end{abstract}

Keywords: triacylglycerol (TAG) compositions; berry seed oils; ultra-performance convergence chromatography (UPC ${ }^{2}$ ); quadrupole time-of-flight mass spectrometry (QTOF MS)

\section{Introduction}

Berries, including cranberry (Vaccinium macrocarpon), raspberry (Rubus idaeus), blackberry (Rubus fruticosus), blueberry (Vaccinium corymbosum) and strawberry (Fragaria $\times$ ananassa), are a group of widely consumed fruits globally. Beside the sweet and sour tastes, berry fruits are well welcomed for particular nutraceutical values in delaying the aging process and reducing the risk of several human chronic diseases [1-3]. To date, these berry fruits are known for their potential antioxidant [4-8], anti-inflammatory [9,10] and antimicrobial properties [5,11-13]. These health benefits enhanced the increasing market demand of berry fruits, but the juicy ovary of berry fruits make them difficult to be transported over long distances. In order to extend the application and increase the shelf life of berry fruits, processed berry products have become more and more popular in recent years. Major berry products, including berry wines, beverages, jams and smoothies, result in large amount of their processing byproducts, 
such as the skin and seeds. The utilizations of the byproducts not only could increase the overall commercial value of berry fruits, but also potentially develop novel food products with high nutritional value.

Berry seeds are known to be rich in minor nutrients and other health beneficial components [14]. These berry seeds are recognized as possible good sources of health-promoting oils due to their great contents of n-3 and total polyunsaturated fatty acids (PUFAs), tocopherols and phytosterols [15-17]. In one study, van Hoed et al. investigated the fatty acid compositions of blackberry, red raspberry, cranberry, blueberry and strawberry seed oils, and reported that the polyunsaturated fatty acids (PUFA) contents were more than $65 \%$ among the overall fatty acids in all the five berry seed oils, with $17.60-36.48 \%$ of $\alpha$-linolenic acid, an n-3 essential fatty acid [18]. In 2014, Radočaj et al. reported PUFA contents of about $76.4 \%$ and $86.2 \%$ for blackberry and raspberry seed oils, respectively [19]. A few other studies also detected high n-3 and total PUFA contents in berry seed oils [16-21]. However, all these previous studies about berry seed oil compositions were focused on their fatty acid composition. None of those previous studies investigated the TAG compositions with sn-location information for the n-3 and other fatty acids. It is known that the sn-position of fatty acids in the TAGs plays a very important role in determining their absorption and bioavailability, and consequently their nutraceutical values [22-25]. For example, the sn-position of palmitic acid in triacylglycerol of milk could significantly change the lipids and calcium absorption, thus affecting fecal conditions of infants [23,24]. Therefore, understanding the sn-positions of the fatty acids is important for their functionality and behavior of the TAG in food systems.

Therefore, the present study was conducted to investigate the TAG compositions of blackberry, red raspberry, black raspberry, blueberry and cranberry seed oils using a supercritical $\mathrm{CO}_{2}$ ultra-performance convergence chromatography $\left(\mathrm{UPC}^{2}\right)$ coupled with high resolution quadrupole time-of-flight mass spectrometry (Q TOF MS). The fatty acid compositions of the five berry seed oils were also determined with a gas chromatography coupled with mass spectrometry (GC-MS) to verify the UPC ${ }^{2}-\mathrm{Q}$ TOF MS results. In addition, the total phenolic contents, as well as the radical scavenging activities of the seed oils, were examined. The results could be used to promote the future nutraceutical development and/or functional food investigation from the seed oils.

\section{Materials and Methods}

\subsection{Materials and Reagents}

Blackberry, red raspberry, black raspberry, blueberry and cranberry seed oil samples were gifted from the Botanic Innovations (Spooner, WI, USA) and stored at $-20^{\circ} \mathrm{C}$ before analyses. All the LC mobile phases and essential chemical agents, including acetonitrile, methanol, isopropanol, and ammonium formate were purchased from Sigma-Aldrich (St. Louis, MO, USA) with a liquid chromatography coupled with mass spectrometry (LC-MS) purity. $\mathrm{CO}_{2}$ was food-grade with purity over $99.99 \%$ and obtained from Zhenxin Gas Co. Ltd. (Shanghai, China). Ultrapure water was purified with a Millipore Milli-Q 10 system (Billerica, MS, USA) in the laboratory. Fatty acid methyl ester (FAME) mixed standard (containing 37 FAMEs) was purchased from NU-CHEK Prep, Inc. (Elysian, MN, USA).

\subsection{Sample Preparations}

All the five seed oil samples were prepared based on our previous published methods [26-29]. One aliquot of $10 \mu \mathrm{L}$ of each berry seed oil sample was mixed with $990 \mu \mathrm{L}$ of acetonitrile/methanol/isopropanol (10:9:1, $v / v / v)$, vortex-mixed for $20 \mathrm{~s}$ and centrifuged at $2000 \mathrm{rpm}$ for $5 \mathrm{~min}$ at an ambient temperature. After removing supernatant, the residue was dissolved in $990 \mu \mathrm{L}$ of isopropanol and injected into UPC ${ }^{2}-\mathrm{Q}$ TOF MS for analysis; each sample was prepared in triplicates. 


\subsection{UPC ${ }^{2}-M S$ System Condition}

The conditions of $\mathrm{UPC}^{2}$-Q TOF MS were set according to a previously reported laboratory procedure [26-29]. Waters Acquity UPC ${ }^{2}$ system (Milford, MA, USA) equipped with an Acquity UPC ${ }^{2}$ BEH HSS C18 column $(150 \mathrm{~mm} \times 3.0 \mathrm{~mm}$ i.d.; $1.7 \mu \mathrm{m})$ was utilized for the separation of TAGs. All the other instrument conditions were similar as previous reported method. A Waters 1525 single pump was used as the compensated pump, and pumped $0.3 \mathrm{~mL} / \mathrm{min}$ of $0.1 \%$ ammonium formate in methanol into the MS source. The TAG compositions of the berry seed oils were analyzed and semi-quantified with a Waters Xevo-G2 Q-TOF MS system as previously described. The ion mode, capillary voltage, cone voltage, temperatures of source and desolvation, as well as other parameters were similar as our previous publications. The collision energy was $6 \mathrm{eV}$ in MS1, and information of fragment ions was collected in the MS2 mode and the collision energy was $35 \mathrm{eV}$, with the scan time set at $0.2 / \mathrm{s}$.

\subsection{Fatty Acid Composition Analysis}

Fatty acid compositions of blackberry, red raspberry, black raspberry, blueberry and cranberry seed oils were determined according to a previously reported laboratory protocol [26-29]. One aliquot of $20 \mathrm{mg}$ of each berry seed oil sample was mixed with $0.4 \mathrm{~mL}$ of methylbenzene and $0.4 \mathrm{~mL}$ of $\mathrm{KOH}-\mathrm{MeOH}(0.5 \mathrm{~mol} / \mathrm{L})$. The mixture was heated at $60{ }^{\circ} \mathrm{C}$ for $10 \mathrm{~min}$ and cooled down to ambient temperature. After that, $2 \mathrm{~mL}$ of boron trifluoride-MeOH (14\%) was added into the mixture that was kept at $60{ }^{\circ} \mathrm{C}$ for $5 \mathrm{~min}$. Then, $2 \mathrm{~mL}$ of isooctane and $3 \mathrm{~mL}$ of ultrapure water were added and vortexed to end the reaction. The supernatant was injected for GC analysis after removing the moisture. The Agilent 7890A gas chromatograph with flame ionization detector (FID) detector and DB-23 silica capillary column ( $60 \mathrm{~m}$ length $\times 0.25 \mathrm{~mm}$ with a $0.25 \mu \mathrm{m}$ film thickness) was used. Fatty acid methyl esters were identified by comparing the retention time of each peak in the berry seed oil samples with that of the FAME standards. And area normalization method was used to calculate the relative concentrations of fatty acids.

\subsection{Total Phenolic Content (TPC) and Radical Scavenging Activity Assays}

The TPC and radical scavenging activity of five berry seed oils were also measured to clarify the potential bioactivity of these oils. In this study, three different radical scavenging assays were utilized to evaluate the radical scavenging activities of berry seed oils, including ABTS radical cation scavenging capacity, relative DPPH radical scavenging capacity (RDSC) and oxygen radical absorbance capacity (ORAC). All the assays were performed following the laboratory protocols previously published [30,31]. In general, $500 \mu \mathrm{L}$ of each berry seed oil was mixed with $500 \mu \mathrm{L}$ of $50 \%$ acetone. After vortexing for $20 \mathrm{~s}$, the mixture was centrifuged at $8000 \mathrm{rpm}$ for $5 \mathrm{~min}$ at an ambient temperature. The $50 \%$ acetone layer was collected, and the oil layer was re-extracted with the same procedure two more times. The three $50 \%$ acetone extractions were combined for further analyses. Each oil sample was extracted in triplicate.

\subsection{Statistical Analysis}

The relative concentrations of TAGs, fatty acids, total phenolic contents and antioxidant activities were reported as the mean \pm standard deviation (SD). One-way ANOVA and Tukey's post hoc test were utilized in SPSS 18.0 (Chicago, IL, USA) to analyze both triacylglycerol and fatty acid compositions, with $p<0.05$ considered a significant difference.

\section{Results and Discussion}

\subsection{Identification of TAGs from the Five Berry Seed Oils}

All the TAGs detected were identified as quasi-molecular ions of $\left[\mathrm{M}+\mathrm{NH}_{4}\right]^{+}$in the Q TOF MS positive ion mode. A total of 52, 53, 52, 59 and 58 TAGs were detected and tentatively identified from the blackberry, red raspberry, black raspberry, blueberry and cranberry seed oils, respectively (Figure 1). The chemical structures of TAGs were 
determined by analyzing their high resolution molecular weight and the mass fragment ion information. Every TAG contains three fatty acids, the initial letter was used to represent a fatty acid, and XYZ represents the structure of a TAG with X, Y and Z fatty acids in its sn-1,2,3 positions. For instance, POL stands for the structure of 1/3-palmitoyl-2-linoleicoyl1/3-oleoylglycerol. Generally, there are three types of TAGs based on the possible fatty acid profiles: three of the same fatty acids such as LLL, two same and one different fatty acids such as OLL, and three different fatty acids such as POL. The chemical structures of all the TAGs were characterized with the elucidation of following three typical TAGs.

(1)

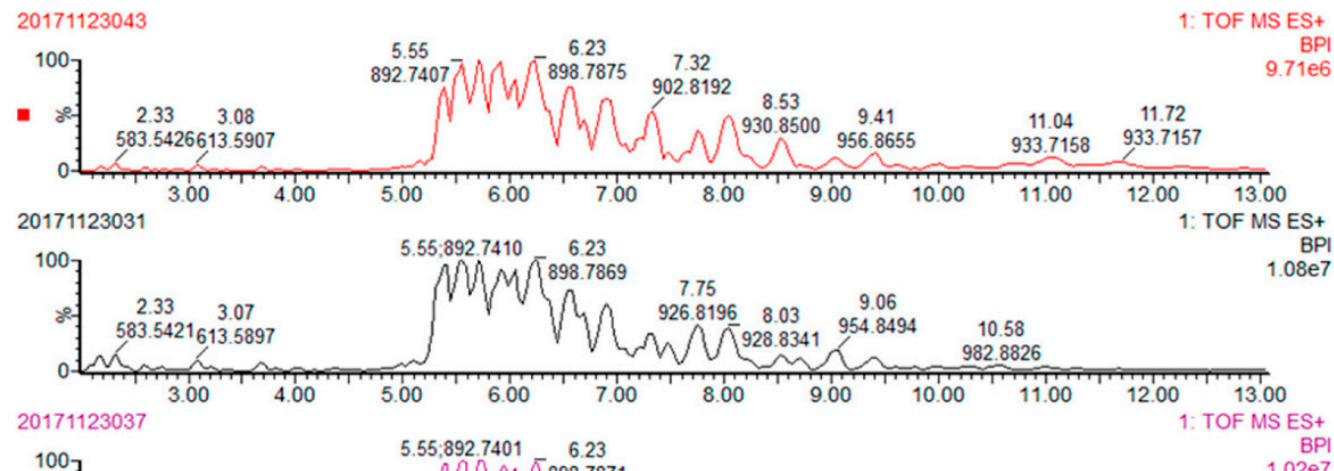

(3)

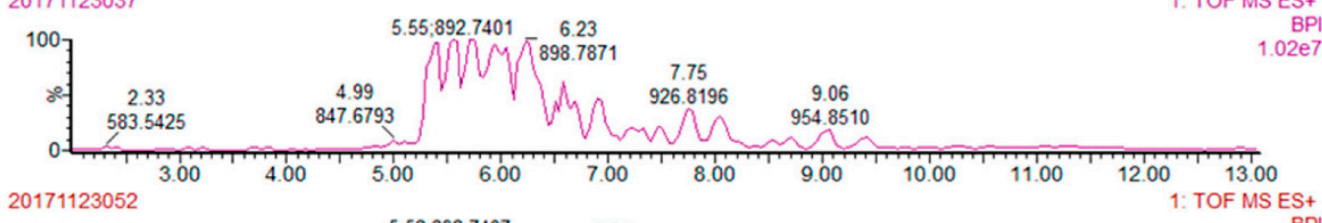

(4)
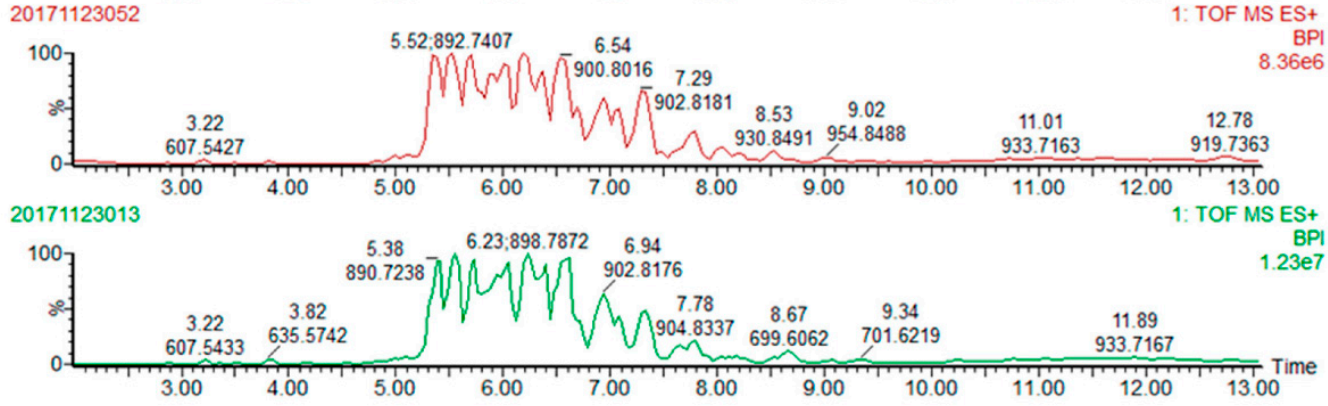

Figure 1. Representative UPC ${ }^{2}-\mathrm{QTOF}$ MS base peak intensity (BPI) chromatograms of the triacylglycerols from (1) blackberry, (2) red raspberry, (3) black raspberry, (4) blueberry and (5) cranberry seed oil samples.

The peak at the retention time $6.94 \mathrm{~min}$ is a representative TAG with similar fatty acids in all the three sn-positions. The quasi-molecular ion of this TAG is $\left[\mathrm{M}+\mathrm{NH}_{4}\right]^{+}$ at $m / z 902.8221$ (Figure 2), the molecular formula could be calculated as $\mathrm{C}_{57} \mathrm{H}_{104} \mathrm{O}_{6}$ based on MS1 information. The MS fragments in its MS2 showed that there was only one fragment ion peak [M-RCOO $+\mathrm{H}]^{+}$at $m / z$ 603.5405, which corresponding to a diacyl fragment $\mathrm{OO}^{+}$ resulted from the TAG eliminating a oleic acid (with the $m / z$ 299.2816). Taken together, this peak could be identified as an OOO TAG.

The peak at the retention time $6.58 \mathrm{~min}$ is an example for identifying TAG with two different fatty acids. The quasi-molecular ion of this TAG is $\left[\mathrm{M}+\mathrm{NH}_{4}\right]^{+}$at $m / z 900.8047$ (Figure 3), the molecular formula was calculated as $\mathrm{C}_{57} \mathrm{H}_{102} \mathrm{O}_{6}$ from the MS1 information. The MS fragment from MS2 showed two major fragment ion peaks $[\mathrm{M}-\mathrm{RCOO}+\mathrm{H}]^{+}$at $m / z 601.5235$ and 603.5349, which correspond to diacyl fragments $\mathrm{LO}^{+}$and $\mathrm{OO}^{+}$from the TAG by eliminating an oleic acid (with the $m / z$ 299.2816) and a linoleic acid (with the $m / z 297.2698$ ), respectively. Due to that the relative natural abundances of $\mathrm{LO}^{+}$and $\mathrm{OO}^{+}$are $100 \%$ and $43 \%$ (Figure 3), and the well accepted knowledge that fatty acid on sn-2 position might have higher bond energy and is harder to be eliminated [29], this TAG is tentatively identified as LOO. 
R-1

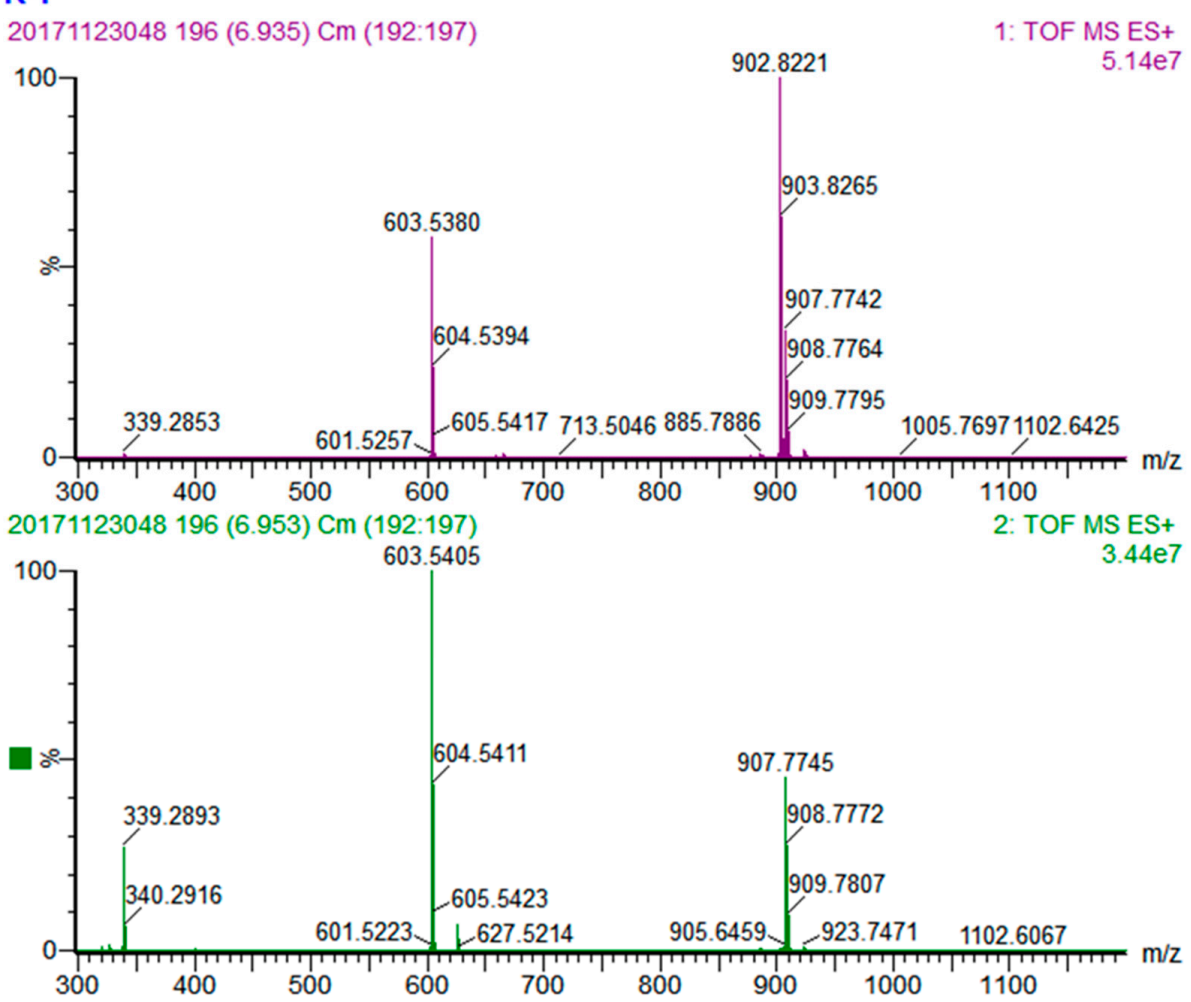

Figure 2. MS spectra of OOO. (Upper) MS1 spectrum and (lower) MS2 spectrum.

R-1
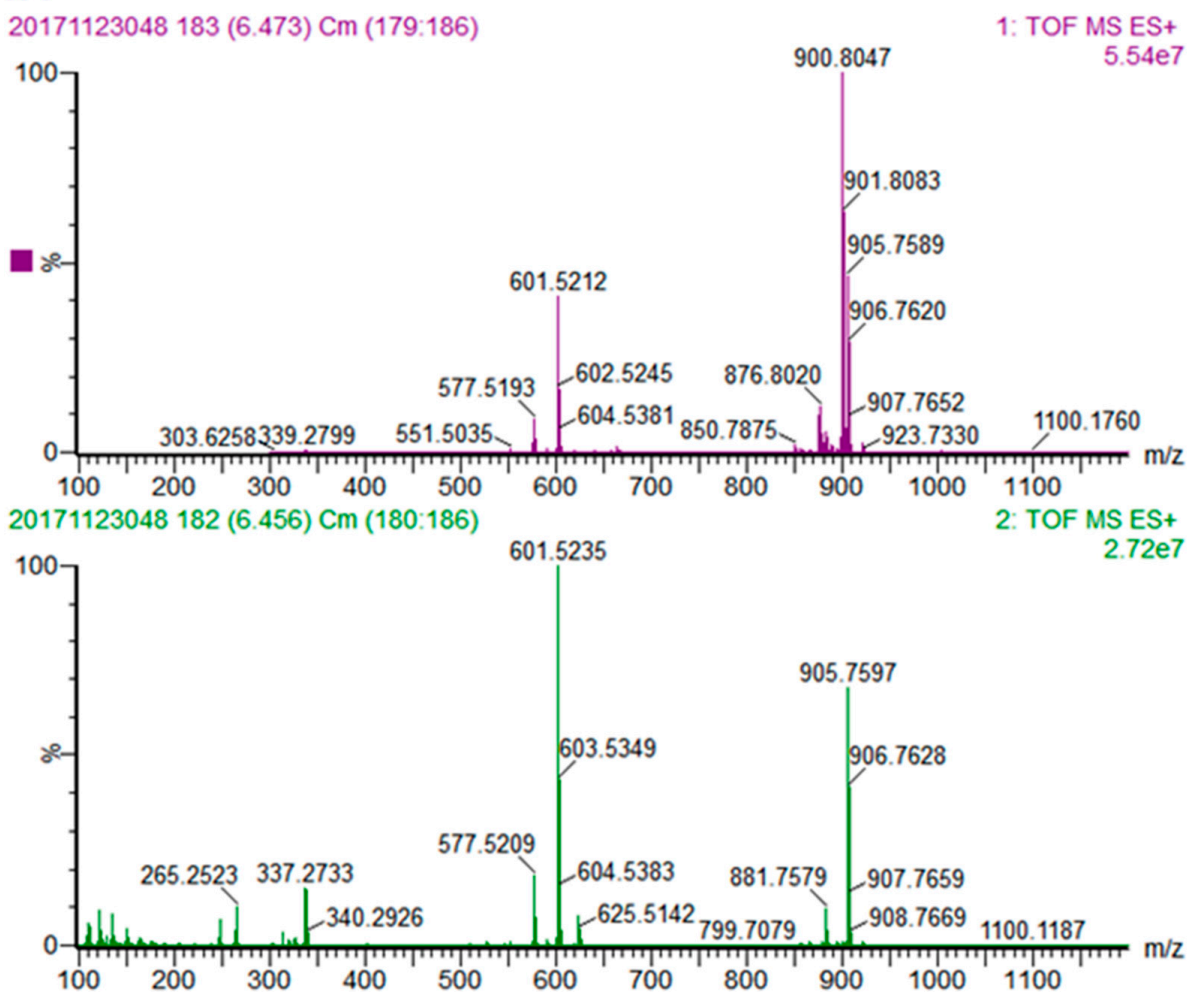

Figure 3. MS spectra of LOO. (Upper) MS1 spectrum and (lower) MS2 spectrum.

The peak with a retention time of $6.30 \mathrm{~min}$ is an example for identification of TAG with three different fatty acids. The quasi-molecular ion of this TAG is $\left[\mathrm{M}+\mathrm{NH}_{4}\right]^{+}$ at $m / z 874.7861$ (Figure 4), and the molecular formula could be calculated as $\mathrm{C}_{55} \mathrm{H}_{100} \mathrm{O}_{6}$ 
based on the MS1 information. MS fragments in the MS2 showed three fragment ion peaks $[\mathrm{M}-\mathrm{RCOO}+\mathrm{H}]^{+}$at $m / z 601.5202,577.5190$ and 575.5037 , respectively. Therefore, the eliminated fatty acid fragments could be calculated as $m / z 273.2659,297.2671$ and 299.2824, which could be identified as palmitic, linoleic and oleic acids, respectively. The three diacyl fragments was identified as $\mathrm{OL}^{+}, \mathrm{PO}^{+}$and $\mathrm{PL}^{+}$, and their natural abundances were $100 \%, 69 \%$ and $46 \%$, respectively. The MS fragment $\mathrm{PL}^{+}$had the lowest natural abundance, suggesting that oleic acid has the highest bond energy and is the most difficult to be eliminated; this TAG was tentatively identified as POL or LOP.

\section{N-2}

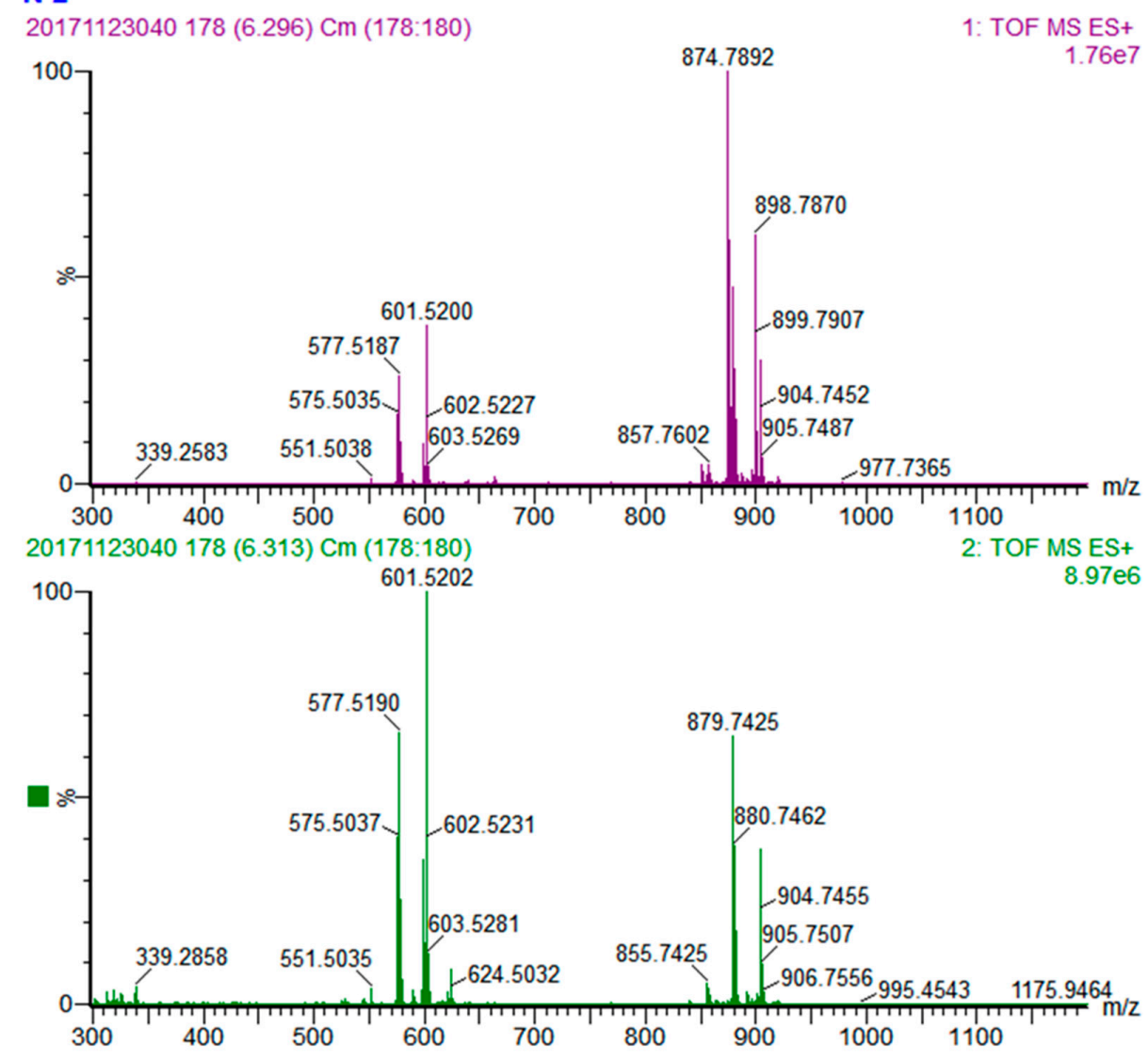

Figure 4. MS spectra of POL. (Upper) MS1 spectrum and (lower) MS2 spectrum.

According to the same rule, the TAG peaks in the five berry seed oils were tentatively identified. Their peak areas were also measured, and the concentrations of each TAG in a berry seed oil were semi-quantified using an area normalization method. The types of TAGs and their relative concentrations in every berry seed oil are discussed separately in the following paragraphs.

There were, in total, 52 TAGs identified from blackberry seed oil. The three primary TAGs in the blackberry seed oil were OLL, LLLn and LnLnL (Table 1), with the relative concentrations at $12.31,8.66$ and $8.51 \mathrm{~g} / 100 \mathrm{~g}$ total TAGs, respectively. Interestingly, similar trends were observed in the TAGs compositions of the red raspberry and black raspberry seed oils. The primary three TAGs in these two raspberry seed oils were also OLL, LLLn and LnLnL, the same as that in the blackberry seed oil. The concentrations of these three TAGs were 14.97, 9.67, and 9.04 g/100 g total TAGs in red raspberry seed oil, and 11.89, 11.67 and $9.95 \mathrm{~g} / 100 \mathrm{~g}$ total TAGs in black raspberry seed oil. These three berry seed oils were similar in their TAG compositions, especially for the TAGs with a concentration more than $5 \mathrm{~g} / 100 \mathrm{~g}$ total TAGs. This might be explained by the fact that the three berries belong to a same Rosaceae family Rubus genus, and with a similar biological botanical relationship. 
Table 1. Identification and relative concentration of triacylglycerols in five berry seed oils.

\begin{tabular}{|c|c|c|c|c|c|c|c|c|c|}
\hline \multirow[b]{2}{*}{$\mathrm{RT}(\mathrm{min})$} & \multirow{2}{*}{$\begin{array}{l}\text { Mass ([M } \\
\left.\left.+\mathrm{NH}_{4}\right]^{+}\right)\end{array}$} & \multirow{2}{*}{$\begin{array}{l}\text { Molecular } \\
\text { Formula }\end{array}$} & \multirow{2}{*}{$\begin{array}{l}\text { Possible } \\
\text { Structure }\end{array}$} & \multirow[b]{2}{*}{ UB } & \multicolumn{5}{|c|}{ TAG Composition (g/100 g TAGs) } \\
\hline & & & & & Blackberry & Red Raspberry & $\begin{array}{c}\text { Black } \\
\text { Raspberry }\end{array}$ & Blueberry & Cranberry \\
\hline 4.46 & 816.7073 & $\mathrm{C}_{51} \mathrm{H}_{90} \mathrm{O}_{6}$ & LaLL & 4 & $0.04 b \pm 0.00$ & $0.06 c \pm 0.01$ & $0.04 b \pm 0.00$ & $0.01 \mathrm{a} \pm 0.00$ & $0.01 \mathrm{a} \pm 0.00$ \\
\hline 4.81 & 840.7066 & $\mathrm{C}_{53} \mathrm{H}_{90} \mathrm{O}_{6}$ & MLnLn & 6 & $0.06 \mathrm{a} \pm 0.01$ & $0.13 b \pm 0.02$ & $0.23 c \pm 0.00$ & $0.09 \mathrm{a} \pm 0.00$ & $0.07 \mathrm{a} \pm 0.00$ \\
\hline 4.99 & 842.7233 & $\mathrm{C}_{53} \mathrm{H}_{92} \mathrm{O}_{6}$ & MLLn & 5 & $0.20 \mathrm{a} \pm 0.02$ & $0.36 \mathrm{c} \pm 0.03$ & $0.44 \mathrm{~d} \pm 0.02$ & $0.27 \mathrm{~b} \pm 0.01$ & $0.20 \mathrm{a} \pm 0.02$ \\
\hline 5.17 & & & MLL & & $0.44 c \pm 0.03$ & $0.43 c \pm 0.01$ & $0.39 \mathrm{~b} \pm 0.01$ & $0.34 \mathrm{~b} \pm 0.02$ & $0.16 \mathrm{a} \pm 0.01$ \\
\hline 5.23 & 844.7394 & $\mathrm{C}_{53} \mathrm{H}_{94} \mathrm{O}_{6}$ & MOLn & 4 & nd & nd & nd & nd & $0.12 \mathrm{a} \pm 0.01$ \\
\hline 5.44 & 846.7556 & $\mathrm{C}_{53} \mathrm{H}_{96} \mathrm{O}_{6}$ & MOL & 3 & $0.04 \mathrm{~b} \pm 0.00$ & $0.01 \mathrm{a} \pm 0.00$ & $0.01 \mathrm{a} \pm 0.00$ & $0.05 \mathrm{~b} \pm 0.00$ & $0.04 \mathrm{~b} \pm 0.00$ \\
\hline 6.05 & 848.7701 & $\mathrm{C}_{53} \mathrm{H}_{98} \mathrm{O}_{6}$ & PLP & 2 & $0.09 \mathrm{~b} \pm 0.00$ & $0.04 \mathrm{a} \pm 0.00$ & $0.03 a \pm 0.00$ & $0.04 \mathrm{a} \pm 0.00$ & $0.03 a \pm 0.00$ \\
\hline 6.40 & 850.7859 & $\mathrm{C}_{53} \mathrm{H}_{100} \mathrm{O}_{6}$ & POP & 1 & $0.07 \mathrm{~b} \pm 0.00$ & $0.03 \mathrm{ab} \pm 0.00$ & $0.01 \mathrm{a} \pm 0.00$ & $0.06 \mathrm{~b} \pm 0.00$ & $0.04 \mathrm{ab} \pm 0.00$ \\
\hline 4.88 & 854.7233 & $\mathrm{C}_{54} \mathrm{H}_{92} \mathrm{O}_{6}$ & PeLLn & 6 & $0.03 \mathrm{a} \pm 0.00$ & $0.09 c \pm 0.01$ & $0.05 \mathrm{~b} \pm 0.00$ & $0.02 \mathrm{a} \pm 0.00$ & $0.06 b \pm 0.00$ \\
\hline 5.23 & & & PoLnL & & $0.62 \mathrm{a} \pm 0.02$ & $0.84 \mathrm{~b} \pm 0.05$ & $0.62 \mathrm{a} \pm 0.03$ & $0.64 \mathrm{a} \pm 0.02$ & $0.56 \mathrm{a} \pm 0.04$ \\
\hline 5.59 & 868.7403 & $\mathrm{C}_{55} \mathrm{H}_{94} \mathrm{O}_{6}$ & PLnLn & 6 & $0.57 c \pm 0.03$ & $0.49 \mathrm{~b} \pm 0.01$ & $0.29 a \pm 0.01$ & $1.29 \mathrm{~d} \pm 0.02$ & $1.75 \mathrm{e} \pm 0.04$ \\
\hline 5.76 & & & PLLn & & $1.08 \mathrm{~b} \pm 0.01$ & $1.02 \mathrm{~b} \pm 0.09$ & $0.73 \mathrm{a} \pm 0.01$ & $1.88 c \pm 0.02$ & $2.21 \mathrm{~d} \pm 0.07$ \\
\hline 5.44 & 870.7551 & $\mathrm{C}_{55} \mathrm{H}_{96} \mathrm{O}_{6}$ & PoLL & 5 & $0.42 c \pm 0.01$ & $0.16 b \pm 0.00$ & $0.09 \mathrm{a} \pm 0.00$ & $0.17 \mathrm{~b} \pm 0.01$ & nd \\
\hline 5.94 & & & PLL & & $2.45 \mathrm{c} \pm 0.04$ & $1.24 \mathrm{a} \pm 0.16$ & $1.19 \mathrm{a} \pm 0.01$ & $1.49 \mathrm{~b} \pm 0.30$ & $1.22 \mathrm{a} \pm 0.08$ \\
\hline 6.08 & 872.7703 & $\mathrm{C}_{55} \mathrm{H}_{98} \mathrm{O}_{6}$ & POLn & & nd & $0.54 \mathrm{~b} \pm 0.00$ & $0.43 a \pm 0.01$ & $1.62 c \pm 0.03$ & $2.05 \mathrm{~d} \pm 0.03$ \\
\hline 6.30 & 874.7861 & $\mathrm{C}_{55} \mathrm{H}_{100} \mathrm{O}_{6}$ & POL & 5 & $1.84 \mathrm{c} \pm 0.04$ & $1.28 \mathrm{~b} \pm 0.08$ & $1.02 \mathrm{a} \pm 0.03$ & $2.09 \mathrm{~d} \pm 0.02$ & $1.79 \mathrm{c} \pm 0.12$ \\
\hline 7.04 & & & LPS & & $0.43 c \pm 0.00$ & $0.17 \mathrm{ab} \pm 0.01$ & $0.13 \mathrm{a} \pm 0.00$ & $0.22 \mathrm{~b} \pm 0.01$ & $0.13 \mathrm{a} \pm 0.00$ \\
\hline 6.65 & 876.8019 & $\mathrm{C}_{55} \mathrm{H}_{102} \mathrm{O}_{6}$ & $\mathrm{POO}$ & 2 & $0.49 \mathrm{~b} \pm 0.01$ & $0.29 \mathrm{a} \pm 0.02$ & $0.18 \mathrm{a} \pm 0.01$ & $1.33 c \pm 0.01$ & $1.51 \mathrm{~d} \pm 0.04$ \\
\hline 7.47 & 878.8129 & $\mathrm{C}_{55} \mathrm{H}_{104} \mathrm{O}_{6}$ & POS & 1 & $0.29 \mathrm{~b} \pm 0.01$ & nd & nd & $0.29 \mathrm{~b} \pm 0.02$ & $0.13 \mathrm{a} \pm 0.01$ \\
\hline 5.33 & 890.7241 & $\mathrm{C}_{55} \mathrm{H}_{96} \mathrm{O}_{6}$ & LnLnLn & 9 & $5.02 \mathrm{a} \pm 0.12$ & $7.36 c \pm 0.19$ & $8.85 \mathrm{~d} \pm 0.21$ & $6.72 b \pm 0.07$ & $6.35 b \pm 0.17$ \\
\hline 5.52 & 892.7397 & $\mathrm{C}_{57} \mathrm{H}_{92} \mathrm{O}_{6}$ & LnLnL & 8 & $8.51 \mathrm{a} \pm 0.12$ & $9.04 \mathrm{ab} \pm 0.74$ & $9.95 b \pm 0.49$ & $8.41 \mathrm{a} \pm 0.30$ & $8.41 \mathrm{a} \pm 0.55$ \\
\hline 5.70 & 894.7553 & $\mathrm{C}_{57} \mathrm{H}_{96} \mathrm{O}_{6}$ & LLLn & 7 & $8.66 b \pm 0.15$ & $9.67 c \pm 0.56$ & $11.67 \mathrm{~d} \pm 0.42$ & $9.71 c \pm 0.11$ & $7.87 a \pm 0.24$ \\
\hline 6.44 & & & LnSLn & & $1.04 \mathrm{a} \pm 0.06$ & $1.45 \mathrm{~b} \pm 0.27$ & $1.43 \mathrm{~b} \pm 0.07$ & $1.08 \mathrm{a} \pm 0.01$ & $0.90 \mathrm{a} \pm 0.06$ \\
\hline 5.91 & 896.7697 & $\mathrm{C}_{57} \mathrm{H}_{98} \mathrm{O}_{6}$ & LLL & 6 & $8.24 c \pm 0.10$ & $6.89 \mathrm{~b} \pm 0.28$ & $9.87 \mathrm{~d} \pm 0.33$ & $4.13 \mathrm{a} \pm 0.01$ & nd \\
\hline 6.05 & & & LOLn & & $4.57 \mathrm{a} \pm 0.27$ & $6.03 \mathrm{bc} \pm 0.32$ & $5.56 \mathrm{~b} \pm 0.37$ & $6.33 c \pm 0.14$ & $10.65 \mathrm{~d} \pm 0.10$ \\
\hline 6.19 & & & OLL & & $12.31 \mathrm{c} \pm 0.24$ & $14.97 \mathrm{~d} \pm 0.42$ & $11.89 c \pm 0.49$ & $8.11 b \pm 0.21$ & $7.37 \mathrm{a} \pm 0.16$ \\
\hline 6.68 & 898.7862 & $\mathrm{C}_{57} \mathrm{H}_{100} \mathrm{O}_{6}$ & LSLn & 5 & $2.75 \mathrm{ab} \pm 0.04$ & $3.58 \mathrm{c} \pm 0.08$ & $3.41 \mathrm{c} \pm 0.35$ & $2.99 \mathrm{~b} \pm 0.02$ & $2.56 \mathrm{a} \pm 0.03$ \\
\hline 6.36 & & & OOLn & & nd & nd & nd & $4.66 \mathrm{a} \pm 0.10$ & $6.08 b \pm 0.38$ \\
\hline 6.58 & & & LOO & & $5.40 \mathrm{a} \pm 0.12$ & $6.79 b \pm 0.13$ & $5.41 \mathrm{a} \pm 0.18$ & $9.54 c \pm 0.14$ & $11.64 \mathrm{~d} \pm 0.27$ \\
\hline 7.07 & 900.8047 & $\mathrm{C}_{57} \mathrm{H}_{102} \mathrm{O}_{6}$ & SOLn & 4 & nd & nd & nd & $3.39 \mathrm{~b} \pm 0.21$ & $2.91 \mathrm{a} \pm 0.15$ \\
\hline 6.9 & & & SLL & & $5.39 \mathrm{~d} \pm 0.09$ & $5.60 \mathrm{~d} \pm 0.20$ & $4.13 c \pm 0.16$ & $3.12 \mathrm{~b} \pm 0.22$ & $2.03 \mathrm{a} \pm 0.00$ \\
\hline 7.32 & & & SOL & & $3.90 \mathrm{~b} \pm 0.09$ & $2.51 \mathrm{a} \pm 0.14$ & nd & $4.26 \mathrm{c} \pm 0.07$ & $4.00 \mathrm{bc} \pm 0.14$ \\
\hline 6.94 & 902.8221 & $\mathrm{C}_{57} \mathrm{H}_{104} \mathrm{O}_{6}$ & OOO & 3 & nd & nd & nd & $4.35 \mathrm{a} \pm 0.11$ & $6.54 b \pm 0.16$ \\
\hline 7.32 & & & SLO & & nd & nd & $1.55 \mathrm{a} \pm 0.16$ & nd & nd \\
\hline 8.21 & & & SSL & & $0.81 c \pm 0.02$ & $0.23 \mathrm{~b} \pm 0.01$ & $0.15 \mathrm{a} \pm 0.00$ & $0.17 \mathrm{a} \pm 0.01$ & nd \\
\hline 7.78 & 904.8322 & $\mathrm{C}_{57} \mathrm{H}_{106} \mathrm{O}_{6}$ & OOS & 2 & $1.18 \mathrm{c} \pm 0.04$ & $0.32 b \pm 0.01$ & $0.18 \mathrm{a} \pm 0.00$ & $2.32 \mathrm{e} \pm 0.03$ & $1.70 \mathrm{~d} \pm 0.03$ \\
\hline 8.74 & 906.8494 & $\mathrm{C}_{57} \mathrm{H}_{108} \mathrm{O}_{6}$ & SSO & 1 & $0.26 \mathrm{c} \pm 0.01$ & $0.05 \mathrm{a} \pm 0.00$ & nd & $0.12 \mathrm{~b} \pm 0.00$ & $0.07 \mathrm{a} \pm 0.00$ \\
\hline 6.79 & & & LLF & & $0.53 c \pm 0.01$ & $0.39 \mathrm{~b} \pm 0.04$ & $0.81 \mathrm{~d} \pm 0.08$ & $0.16 \mathrm{a} \pm 0.01$ & $0.43 \mathrm{bc} \pm 0.03$ \\
\hline 7.00 & 924.8030 & $\mathrm{C}_{59} \mathrm{H}_{108} \mathrm{O}_{6}$ & LnLG & & $0.61 b \pm 0.04$ & $0.69 \mathrm{~b} \pm 0.03$ & $1.30 \mathrm{c} \pm 0.07$ & $0.38 a \pm 0.00$ & $0.61 b \pm 0.03$ \\
\hline 7.47 & & & LnLnA & & $1.06 \mathrm{c} \pm 0.02$ & $1.65 \mathrm{~d} \pm 0.13$ & $1.67 \mathrm{~d} \pm 0.10$ & $0.72 \mathrm{~b} \pm 0.01$ & $0.33 a \pm 0.03$ \\
\hline 7.22 & & & LLG & & $2.29 \mathrm{~d} \pm 0.13$ & $1.23 \mathrm{~b} \pm 0.12$ & $1.78 c \pm 0.04$ & $0.69 \mathrm{a} \pm 0.06$ & $1.31 b \pm 0.05$ \\
\hline 7.39 & 926.8173 & $\mathrm{C}_{59} \mathrm{H}_{104} \mathrm{O}_{6}$ & LnOG & 5 & nd & nd & nd & $0.30 \mathrm{a} \pm 0.03$ & $0.71 b \pm 0.04$ \\
\hline 7.75 & & & LnLA & & $2.75 c \pm 0.10$ & $3.30 \mathrm{~d} \pm 0.28$ & $3.37 \mathrm{~d} \pm 0.31$ & $1.12 \mathrm{~b} \pm 0.01$ & $0.68 \mathrm{a} \pm 0.01$ \\
\hline 8.21 & & & LnOA & & nd & nd & nd & $0.57 b \pm 0.03$ & $0.42 \mathrm{a} \pm 0.03$ \\
\hline 7.64 & 928.8326 & $\mathrm{C}_{59} \mathrm{H}_{106} \mathrm{O}_{6}$ & LOG & 4 & $1.59 \mathrm{~b} \pm 0.11$ & $1.02 \mathrm{a} \pm 0.08$ & $1.04 \mathrm{a} \pm 0.12$ & $0.97 \mathrm{a} \pm 0.04$ & $1.59 \mathrm{~b} \pm 0.07$ \\
\hline 8.03 & & & LLA & & $5.11 \mathrm{e} \pm 0.05$ & $3.35 \mathrm{~d} \pm 0.20$ & $3.09 \mathrm{c} \pm 0.37$ & $0.94 \mathrm{~b} \pm 0.03$ & $0.48 \mathrm{a} \pm 0.03$ \\
\hline 8.10 & & & OOG & & nd & nd & nd & nd & $0.62 \mathrm{a} \pm 0.01$ \\
\hline 8.53 & 930.848 & $\mathrm{C}_{59} \mathrm{H}_{108} \mathrm{O}_{6}$ & LOA & 3 & $2.25 \mathrm{~d} \pm 0.04$ & $1.06 \mathrm{c} \pm 0.10$ & $0.83 \mathrm{~b} \pm 0.03$ & $0.87 b \pm 0.06$ & $0.51 \mathrm{a} \pm 0.01$ \\
\hline 9.06 & & & $\mathrm{OOA}$ & & $0.60 \mathrm{~d} \pm 0.02$ & $0.13 \mathrm{ab} \pm 0.01$ & $0.09 \mathrm{a} \pm 0.00$ & $0.24 c \pm 0.00$ & $0.18 \mathrm{~b} \pm 0.00$ \\
\hline 9.63 & 932.8641 & $\mathrm{C}_{59} \mathrm{H}_{110} \mathrm{O}_{6}$ & LSA & 2 & $0.49 \mathrm{~d} \pm 0.02$ & $0.12 b \pm 0.01$ & $0.09 \mathrm{~b} \pm 0.00$ & $0.03 \mathrm{a} \pm 0.00$ & $0.02 \mathrm{a} \pm 0.00$ \\
\hline 8.71 & & & OLHo & & nd & nd & nd & $0.11 \mathrm{~b} \pm 0.00$ & $0.05 \mathrm{a} \pm 0.00$ \\
\hline 8.67 & 942.8499 & $\mathrm{C}_{60} \mathrm{H}_{108} \mathrm{O}_{6}$ & LLHn & 4 & $0.33 \mathrm{a} \pm 0.01$ & $0.31 \mathrm{a} \pm 0.03$ & $0.31 \mathrm{a} \pm 0.01$ & nd & nd \\
\hline 8.71 & 952.8345 & $\mathrm{C}_{61} \mathrm{H}_{106} \mathrm{O}_{6}$ & LnLnB & 6 & $0.46 c \pm 0.01$ & $0.87 \mathrm{~d} \pm 0.08$ & $1.03 \mathrm{e} \pm 0.09$ & $0.32 \mathrm{~b} \pm 0.02$ & $0.14 \mathrm{a} \pm 0.00$ \\
\hline 9.02 & & & LnLB & & $1.11 c \pm 0.05$ & $1.41 \mathrm{~d} \pm 0.17$ & $1.65 \mathrm{~d} \pm 0.23$ & $0.47 \mathrm{~b} \pm 0.02$ & $0.20 a \pm 0.01$ \\
\hline 9.37 & & & LLB & & $1.59 \mathrm{e} \pm 0.02$ & $0.93 c \pm 0.12$ & $1.13 \mathrm{~d} \pm 0.13$ & $0.24 \mathrm{~b} \pm 0.01$ & $0.12 \mathrm{a} \pm 0.00$ \\
\hline 9.59 & 956.864 & $\mathrm{C}_{61} \mathrm{H}_{110} \mathrm{O}_{6}$ & $\begin{array}{l}\text { LnOB } \\
\text { LnOB }\end{array}$ & 4 & nd & $0.23 b \pm 0.03$ & $0.23 b \pm 0.00$ & $0.13 \mathrm{a} \pm 0.00$ & $0.09 a \pm 0.00$ \\
\hline 9.95 & 958.8807 & $\mathrm{C}_{61} \mathrm{H}_{112} \mathrm{O}_{6}$ & LOB & 3 & $0.58 \mathrm{~d} \pm 0.02$ & $0.32 \mathrm{c} \pm 0.04$ & $0.28 \mathrm{c} \pm 0.01$ & $0.15 b \pm 0.00$ & $0.08 \mathrm{a} \pm 0.00$ \\
\hline 10.65 & 960.8965 & $\mathrm{C}_{61} \mathrm{H}_{114} \mathrm{O}_{6}$ & LSB & 2 & $0.16 b \pm 0.01$ & $0.05 \mathrm{a} \pm 0.00$ & $0.04 \mathrm{a} \pm 0.00$ & $0.04 \mathrm{a} \pm 0.00$ & $0.04 \mathrm{a} \pm 0.00$ \\
\hline 10.16 & 970.8806 & $\mathrm{C}_{62} \mathrm{H}_{112} \mathrm{O}_{6}$ & LLT & 4 & $0.33 c \pm 0.01$ & $0.19 \mathrm{~b} \pm 0.02$ & $0.22 \mathrm{~b} \pm 0.01$ & $0.05 \mathrm{a} \pm 0.00$ & $0.03 a \pm 0.00$ \\
\hline 10.16 & 980.8647 & $\mathrm{C}_{63} \mathrm{H}_{110} \mathrm{O}_{6}$ & LnLnLi & 6 & $0.17 \mathrm{~b} \pm 0.01$ & $0.25 c \pm 0.02$ & $0.22 c \pm 0.00$ & $0.07 \mathrm{a} \pm 0.00$ & $0.07 a \pm 0.00$ \\
\hline 10.54 & 982.8818 & $\mathrm{C}_{63} \mathrm{H}_{112} \mathrm{O}_{6}$ & LnLLi & 5 & $0.30 \mathrm{~b} \pm 0.01$ & $0.43 c \pm 0.02$ & $0.43 c \pm 0.01$ & $0.07 \mathrm{a} \pm 0.00$ & $0.08 \mathrm{a} \pm 0.00$ \\
\hline 11.01 & 984.8955 & $\mathrm{C}_{63} \mathrm{H}_{114} \mathrm{O}_{6}$ & LLLi & 4 & $0.43 c \pm 0.01$ & $0.33 \mathrm{~b} \pm 0.02$ & $0.39 \mathrm{bc} \pm 0.01$ & $0.04 \mathrm{a} \pm 0.00$ & $0.04 \mathrm{a} \pm 0.00$ \\
\hline 12.42 & 1010.9115 & $\mathrm{C}_{65} \mathrm{H}_{116} \mathrm{O}_{6}$ & LnLH & 5 & $0.03 \mathrm{~b} \pm 0.00$ & $0.09 \mathrm{c} \pm 0.01$ & $0.09 c \pm 0.00$ & $0.01 \mathrm{a} \pm 0.00$ & $0.02 \mathrm{a} \pm 0.00$ \\
\hline
\end{tabular}

TAGs, triacylglycerols; RT, retention time; UB, number of unsaturation; nd, not detectable; $\mathrm{P}$, palmitic acid; M, myristic acid; O, oleic acid; S, stearic acid; L, linoleic acid; Ln, linolenic acid; G, gondoic acid; A, arachidic acid; B, behenic acid. X-X-Y, X-Y-X, X-X-X and X-Y-Z represent the structures of triacylglycerols; for example, SPO stands for the structure of 1/3-stearoyl-2-palmitoyl-1/3-oleoylglycerol. The relative concentration of each triacylglycerol is reported as gram of triacylglycerols/100 g of oil samples. Cranberry, red raspberry, black raspberry, blackberry and blueberry seed oils were analyzed in triplicates and results reported as mean \pm standard deviation (SD). Different letters represent significant differences within a column $(p<0.05)$.

A total of 59 TAGs were identified from the blueberry seed oil. Among all the TAGs, LLLn, LOO and LnLnL were the three primary ones, with relative concentrations at 9.71, 9.54 and $8.41 \mathrm{~g} / 100 \mathrm{~g}$ total TAGs, respectively. For cranberry seed oil, 58 TAGs were separated and 
identified, and LOO (11.64 g/100 g), LOLn (10.65 g/100 g) and LnLnL (8.41 g/100 g) were the three greatest TAGs.

Since every TAG has three bounded fatty acids, the compositions of all the TAGs could be used to calculate the fatty acid compositions in berry seed oils. Based on the present TAG composition results, linoleic acid and linolenic acid are the two primary fatty acids in all the five berry seed oils. Linoleic acid concentrations were more than $50 \mathrm{~g} / 100 \mathrm{~g}$ total TAGs in blackberry, red raspberry and black raspberry seed oils, and more than $30 \mathrm{~g} / 100 \mathrm{~g}$ total TAGs in the blueberry and cranberry seed oils. It is important that $\alpha$-linolenic acid levels were more than $30 \mathrm{~g} / 100 \mathrm{~g}$ total TAGs in all the five berry seed oils, suggesting that these berry seed oils may serve as excellent dietary sources for this n-3 essential fatty acid and may be included in human diet for improving the $n-6 / n-3$ ratio. These phenomena showed that all these berry seed oils contained great amounts of multi-unsaturated fatty acids (MUFA), which have potential abilities to be developed for food supplements or nutrients.

\subsection{Identifications of Fatty Acids from Five Berry Seed Oils}

The fatty acid compositions of five berry seed oils were also directly examined using GC-MS, and five major fatty acids, including palmitic (16:0), stearic (18:0), oleic (18:1), linoleic (18:2) and linolenic (18:3) acids were identified and semi-quantified (Table 2). All the five berry seed oils contained over $90 \%$ of unsaturated fatty acids on a per weight basis, and less than $10 \%$ of saturated fatty acids. Among the saturated fatty acids, palmitic acid content was 2-3 times greater than that of stearic acid in all the five berry seed oils. Linoleic acid content was the greatest among all unsaturated fatty acids and accounted for over $50 \%$ of the total fatty acids in the blackberry, red raspberry and blackberry seed oils, in excellent agreement to the fatty acid profiles calculated from the TAG compositions (Table 2). These fatty acid compositions also agreed to that of the blackberry and raspberry seed oils reported previously [18-20,32]. In the present study, $\alpha$-linolenic acid had the greatest concentration and consisted of about $50 \%$ of the total fatty acids in the blueberry and cranberry seed oils, followed by linoleic acid at a level of about $40 \mathrm{~g} / 100 \mathrm{~g}$ total fatty acids. The fatty acid results obtained by GC-MS analysis supported the fatty acid compositions calculated from the TAGs (Table 2). It is understandable that minor amounts of fatty acids identified in the TAGs cannot be detected by GC-MS due to the detection limits of the two methods, since the limit of detection of fatty acids in the GC method are generally at microgram per milliliter, which is much higher than that in $\mathrm{UPC}^{2}$-MS. The sample preparation might also contribute to the different fatty acid profile data by GC-MS and $\mathrm{UPC}^{2}$-MS.

\subsection{Total Phenolic Contents and Radical Scavenging Activities of Five Berry Seed Oils}

The total phenolic contents and radical scavenging activities of the five berry seed oils were investigated in this study (Figure 5). The seed oils differed in their TPC values, ranging from 13.68 to $177.06 \mu \mathrm{mol}$ GAE (gallic acid equivalent)/L oil. Red raspberry seed oil showed the greatest TPC value, followed by black raspberry seed oil at $68.62 \mu \mathrm{mol}$ GAE/L oil, blackberry seed oil at $52.72 \mu \mathrm{mol}$ GAE/L oil, blueberry seed oil at $24.16 \mu \mathrm{mol}$ GAE/L oil and cranberry seed oil. The red raspberry seed oil was the most rich in phenolic compounds under the experimental conditions.

Different radical scavenging activity assays could be used to evaluate the radical scavenging abilities of a sample against different types of free radicals. In general, two or more assays are needed to draw a common conclusion for a radical scavenging sample because of the possible interference of the assay system. In this study, three different assays were applied to determine the radical scavenging activities of berry seed oils. Generally, all the tested berry seed oil samples showed the greatest abilities in competitively inhibiting oxygen radicals, since the ORAC values for all the berry seed oils were much greater than the results of other two assays. The blackberry seed oil showed the greatest radical scavenging activity, followed by red raspberry in all three assays. The blueberry and blackberry seed oils showed greater $\mathrm{ABTS}^{\bullet+}$ and $\mathrm{DPPH}^{\bullet}$ scavenging activities than the cranberry seed oil, but their ORAC values were smaller than that of the cranberry seed oil. 
These results indicated that different berry seed oils might contain different antioxidant capabilities and differed in their interactions with individual radical systems.

Table 2. The fatty acid compositions in the five berry seed oils.

\begin{tabular}{ccccccc}
\hline \multirow{2}{*}{ Fatty Acid } & C:D & \multicolumn{5}{c}{ Fatty Acid Composition (g/100 g Total FAs) } \\
\cline { 3 - 7 } & & Blackberry & Red Raspberry & Black Raspberry & Blueberry & Cranberry \\
\hline palmitic acid & $16: 0$ & $5.63 \mathrm{c} \pm 0.03$ & $3.70 \mathrm{~b} \pm 0.08$ & $2.40 \mathrm{a} \pm 0.04$ & $7.47 \mathrm{~d} \pm 0.10$ & $7.68 \mathrm{~d} \pm 0.02$ \\
stearic acid & $18: 0$ & $2.81 \mathrm{~d} \pm 0.02$ & $1.39 \mathrm{~b} \pm 0.06$ & $0.84 \mathrm{a} \pm 0.01$ & $2.38 \mathrm{c} \pm 0.05$ & $1.56 \mathrm{~b} \pm 0.03$ \\
oleic acid & $18: 1$ & $1.34 \mathrm{a} \pm 0.06$ & $1.36 \mathrm{a} \pm 0.14$ & $1.17 \mathrm{a} \pm 0.04$ & $1.15 \mathrm{a} \pm 0.09$ & $1.13 \mathrm{a} \pm 0.04$ \\
linoleic acid & $18: 2$ & $59.12 \mathrm{f} \pm 0.26$ & $52.37 \mathrm{e} \pm 0.30$ & $51.03 \mathrm{~d} \pm 0.28$ & $40.20 \mathrm{c} \pm 0.12$ & $38.40 \mathrm{~b} \pm 0.16$ \\
linolenic acid & $18: 3$ & $31.10 \mathrm{a} \pm 0.28$ & $41.18 \mathrm{~b} \pm 0.51$ & $44.56 \mathrm{c} \pm 0.33$ & $48.80 \mathrm{~d} \pm 0.34$ & $51.23 \mathrm{e} \pm 0.16$ \\
SFA & & 8.44 & 5.09 & 3.24 & 9.85 & 9.24 \\
UFA & & 91.56 & 94.91 & 96.76 & 90.15 & 90.76 \\
MUFA & & 1.34 & 1.36 & 1.17 & 89.15 & 1.13 \\
PUFA & & 90.22 & 93.55 & 95.59 & 89.00 & 89.63
\end{tabular}

FAs, fatty acids; C:D, carbon number: double bounds number; nd, not detectable; SFA, saturated fatty acids UFA, total unsaturated fatty acids; MUFA, monounsaturated fatty acids; PUFA, polyunsaturated fatty acids. The relative concentration of each fatty acid is reported as gram of fatty acids $/ 100 \mathrm{~g}$ of total fatty acids. All the seed oils were analyzed in triplicates and results reported as mean \pm standard deviation (SD). Different letters represent significant differences within a column $(p<0.05)$.
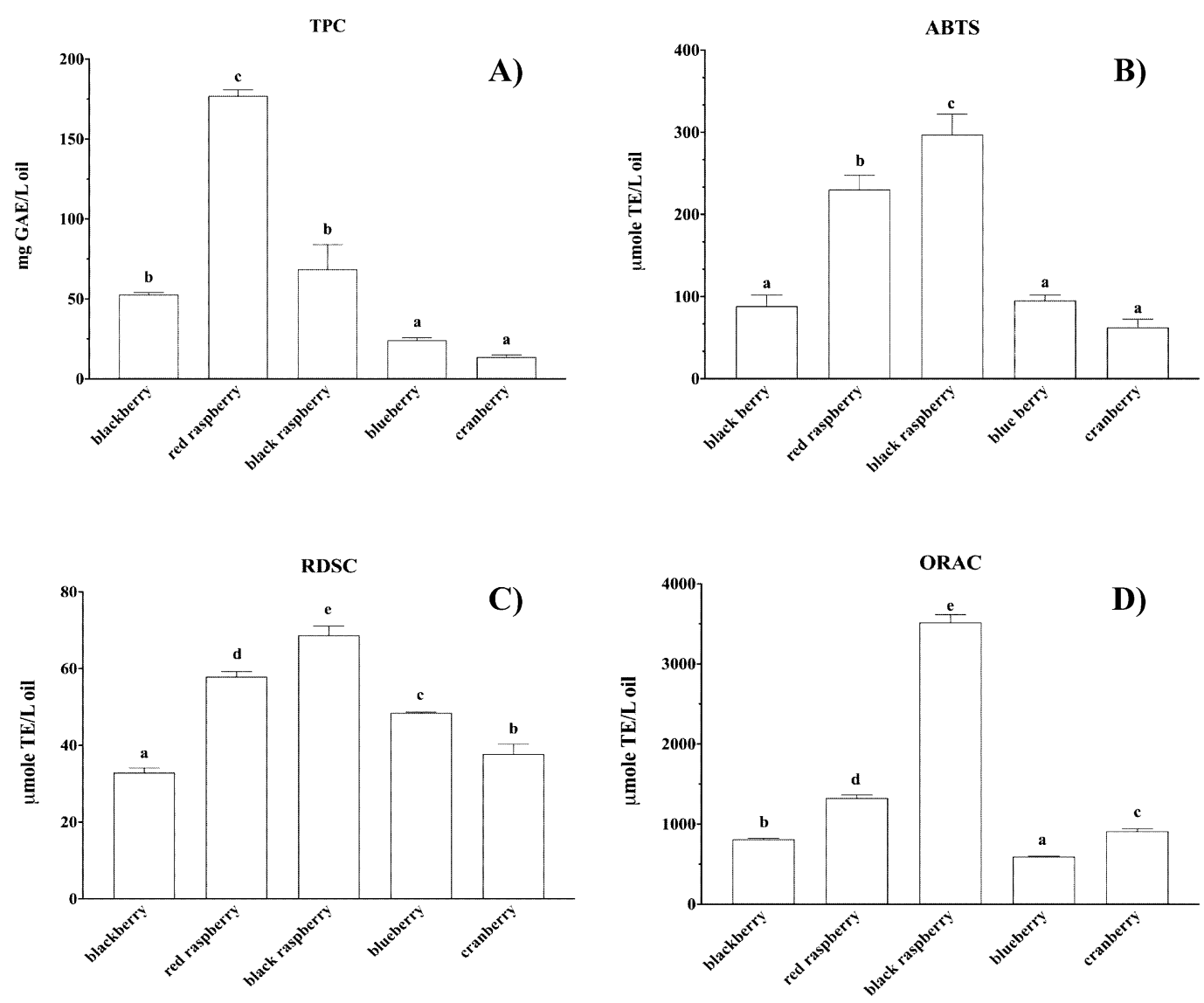

Figure 5. (A) Total phenolic content (TPC), (B) ABTS radical cation scavenging capacity, (C) relative DPPH radical scavenging capacity (RDSC), and (D) oxygen radical absorbance capacity (ORAC) of the blackberry, red raspberry, black raspberry, blueberry and cranberry seed oil extracts. Different letters represent significant differences within a column $(p<0.05)$.

\section{Conclusions}

In summary, this study examined the TAG compositions and the sn-positions of individual fatty acids of the five berry seed oils for the first time. Linoleic $(18: 2 n-6)$ and $\alpha$-linolenic (18:3n-3) acids, the two essential PUFAs, accounted for more than $90 \%$ of the total fatty acids in the berry seed oils with a much greater $n-3 / n-6$ ratio than most of the commonly consumed vegetable oils. In addition, the seed oils are rich in natural 
antioxidants. The results from this study may provide a scientific basis for improved utilization of these seed oils for human nutrition and health, especially important for their potential in dietary intake of bioavailable $\alpha$-linolenic acid and improving the balance between dietary n-3 and n-6 PUFAs. Further animal and pilot human studies could be designed to investigate the nutritional values and health benefits of these berry seed oils in the future.

Author Contributions: Conceptualization, B.G. and Y.L. (Yanfang Li); methodology, Y.L. (Yinghua Luo); software, Y.L. (Yinghua Luo) and F.Y.; validation, J.W., B.G. and F.Y.; investigation, Y.L. (Yinghua Luo); data curation, Y.L. (Yanfang Li) and F.Y.; writing —original draft preparation, Y.L. (Yinghua Luo); writing-review and editing, B.G.; supervision, L.Y.; project administration, L.Y.; funding acquisition, J.W. and B.G. All authors have read and agreed to the published version of the manuscript.

Funding: This research was funded by the National Natural Science Foundation of China (Grant No. 32001819, 32001815).

Institutional Review Board Statement: Not applicable.

Informed Consent Statement: Not applicable.

Data Availability Statement: Not applicable.

Conflicts of Interest: The authors declare no conflict of interest.

\section{References}

1. Ferreira, D.; Gross, G.; Kolodziej, H.; Yoshida, T. Tannins and related polyphenols: Fascinating natural products with diverse implications for biological systems, ecology, industrial applications and health protection. Phytochemistry 2005, 66, 1969-1971. [CrossRef] [PubMed]

2. La Vecchia, C.; Altieri, A.; Tavani, A. Vegetables, fruit, antioxidants and cancer: A review of Italian studies. Eur. J. Nutr. 2001, 40, 261-267. [CrossRef] [PubMed]

3. Yao, L.; Jiang, Y.; Shi, J.; Tomas-Barberan, F.; Datta, N.; Singanusong, R.; Chen, S. Flavonoids in food and their health benefits. Plant Foods Hum. Nutr. 2004, 59, 113-122. [CrossRef] [PubMed]

4. Heinonen, I.; Meyer, A.; Frankel, E. Antioxidant activity of berry phenolics on human low-density lipoprotein and liposome oxidation. J. Agric. Food Chem. 1998, 46, 4107-4112. [CrossRef]

5. Heinonen, M. Antioxidant activity and antimicrobial effect of berry phenolics-a Finnish perspective. Mol. Nutr. Food Res. 2007, 51, 684-691. [CrossRef]

6. Kähkönen, M.P.; Hopia, A.I.; Heinonen, M. Berry phenolics and their antioxidant activity. J. Agric. Food Chem. 2001, 49, 4076-4082. [CrossRef]

7. Scalzo, J.; Mezzetti, B.; Battino, M. Total antioxidant capacity evaluation: Critical steps for assaying berry antioxidant features. Biofactors 2005, 23, 221-227. [CrossRef] [PubMed]

8. Zafra-Stone, S.; Yasmin, T.; Bagchi, M.; Chatterjee, A.; Vinson, J.A.; Bagchi, D. Berry anthocyanins as novel antioxidants in human health and disease prevention. Mol. Nutr. Food Res. 2007, 51, 675-683. [CrossRef] [PubMed]

9. Jensen, G.S.; Wu, X.; Patterson, K.M.; Barnes, J.; Carter, S.G.; Scherwitz, L.; Beaman, R.; Endres, J.R.; Schauss, A.G. In vitro and in vivo antioxidant and anti-inflammatory capacities of an antioxidant-rich fruit and berry juice blend. Results of a pilot and randomized, double-blinded, placebo-controlled, crossover study. J. Agric. Food Chem. 2008, 56, 8326-8333. [CrossRef] [PubMed]

10. Nardi, G.M.; Farias Januario, A.G.; Freire, C.G.; Megiolaro, F.; Schneider, K.; Perazzoli, M.R.; Locatelli, C. Anti-inflammatory Activity of Berry Fruits in Mice Model of Inflammation is Based on Oxidative Stress Modulation. Pharmacogn. Res. 2016, 8, 42-49. [CrossRef]

11. López de Dicastillo, C.; Bustos, F.; Valenzuela, X.; López-Carballo, G.; Vilariño, J.M.; Galotto, M.J. Chilean berry Ugni molinae Turcz. fruit and leaves extracts with interesting antioxidant, antimicrobial and tyrosinase inhibitory properties. Food Res. Int. 2017, 102, 119-128. [CrossRef]

12. Nohynek, L.J.; Alakomi, H.L.; Kähkönen, M.P.; Heinonen, M.; Helander, I.M.; Oksman-Caldentey, K.M.; Puupponen-Pimiä, R.H. Berry phenolics: Antimicrobial properties and mechanisms of action against severe human pathogens. Nutr. Cancer 2006, 54, 18-32. [CrossRef]

13. Puupponen-Pimiä, R.; Nohynek, L.; Meier, C.; Kähkönen, M.; Heinonen, M.; Hopia, A.; Oksman-Caldentey, K.M. Antimicrobial properties of phenolic compounds from berries. J. Appl. Microbiol. 2001, 90, 494-507. [CrossRef]

14. Shahidi, F. Antioxidant factors in plant foods and selected oilseeds. Biofactors 2000, 13, 179-185. [CrossRef]

15. Helbig, D.; Bohm, V.; Wagner, A.; Schubert, R.; Jahreis, G. Berry seed press residues and their valuable ingredients with special regard to black currant seed press residues. Food Chem. 2008, 111, 1043-1049. [CrossRef]

16. Kallio, H.; Yang, B.; Peippo, P.; Tahvonen, R.; Pan, R. Triacylglycerols, glycerophospholipids, tocopherols, and tocotrienols in berries and seeds of two subspecies (ssp. sinensis and mongolica) of Sea Buckthorn (Hippophä̈ rhamnoides). J. Agric. Food Chem. 2002, 50, 3004-3009. [CrossRef] 
17. Yang, B.; Karlsson, R.M.; Oksman, P.H.; Kallio, H.P. Phytosterols in sea buckthorn (Hippophaë rhamnoides L.) berries: Identification and effects of different origins and harvesting times. J. Agric. Food Chem. 2001, 49, 5620-5629. [CrossRef]

18. Van Hoed, V.; De Clercq, N.; Echim, C.; Andjelkovic, M.; Leber, E.; Dewettinck, K.; Verhe, R. Berry seeds: A source of specialty oils with high content of bioactives and nutritional. J. Food Lipids 2009, 16, 33-49. [CrossRef]

19. Radocaj, O.; Vujasinovic, V.; Dimic, E.; Basic, Z. Blackberry (Rubus fruticosus L.) and raspberry (Rubus idaeus L.) seed oils extracted from dried press pomace after longterm frozen storage of berries can be used as functional food ingredients. Eur. J. Lipid Sci. Technol. 2014, 116, 1015-1024. [CrossRef]

20. Parry, J.; Su, L.; Luther, M.; Zhou, K.; Yurawecz, M.P.; Whittaker, P.; Yu, L. Fatty acid composition and antioxidant properties of cold-pressed marionberry, boysenberry, red raspberry, and blueberry seed oils. J. Agric. Food Chem. 2005, 53, 566-573. [CrossRef]

21. Van Hoed, V.; Barbouche, I.; De Clercq, N.; Dewettinck, K.; Slah, M.; Leber, E.; Verhe, R. Influence of filtering of cold pressed berry seed oils on their antioxidant profile and quality characteristics. Food Chem. 2011, 127, 1848-1855. [CrossRef]

22. Guo, Y.; Cai, Z.; Xie, Y.; Ma, A.; Zhang, H.; Rao, P.; Wang, Q. Synthesis, physicochemical properties, and health aspects of structured lipids: A review. Compr. Rev. Food Sci. Food Saf. 2020, 19, 759-800. [CrossRef]

23. Wan, J.; Hu, S.; Ni, K.; Chang, G.; Sun, X.; Yu, L. Characterisation of Fecal Soap Fatty Acids, Calcium Contents, Bacterial Community and Short-Chain Fatty Acids in Sprague Dawley Rats Fed with Different sn-2 Palmitic Triacylglycerols Diets. PLoS ONE 2016, 11, e0164894. [CrossRef]

24. Yang, P.; Zhang, H.; Wan, J.; Hu, J.; Liu, J.; Wang, J.; Yu, L. Dietary sn-2 palmitic triacylglycerols reduced faecal lipids, calcium contents and altered lipid metabolism in Sprague-Dawley rats. Int. J. Food Sci. Nutr. 2019, 70, 474-483. [CrossRef]

25. Yoshinaga, K.; Sasaki, K.; Watanabe, H.; Nagao, K.; Inoue, N.; Shirouchi, B.; Gotoh, N. Differential effects of triacylglycerol positional isomers containing n-3 series highly unsaturated fatty acids on lipid metabolism in C57BL/6J mice. J. Nutr. Biochem. 2015, 26, 57-63. [CrossRef]

26. Gao, B.; Luo, Y.; Lu, W.; Liu, J.; Zhang, Y.; Yu, L.L. Triacylglycerol compositions of sunflower, corn and soybean oils examined with supercritical $\mathrm{CO}^{2}$ ultra-performance convergence chromatography combined with quadrupole time-of-flight mass spectrometry. Food Chem. 2017, 218, 569-574. [CrossRef]

27. Li, Y.; Yuan, F.; Wu, Y.; Zhang, Y.; Gao, B.; Yu, L. Triacylglycerols and Fatty Acid Compositions of Cucumber, Tomato, Pumpkin, and Carrot Seed Oils by Ultra-Performance Convergence Chromatography Combined with Quadrupole Time-of-Flight Mass Spectrometry. Foods 2020, 9, 970. [CrossRef]

28. Luo, Y.; Zhang, Y.; Yuan, F.; Gao, B.; Wang, Z.; Yu, L. Triacylglycerols composition analysis of olive oils by ultra-performance convergence chromatography combined with quadrupole time-of-flight mass spectrometry. Int. J. Food Sci. Technol. 2019, 54, 871-879. [CrossRef]

29. Zhou, Q.; Gao, B.; Zhang, X.; Xu, Y.; Shi, H.; Yu, L. Chemical profiling of triacylglycerols and diacylglycerols in cow milk fat by ultra-performance convergence chromatography combined with a quadrupole time-of-flight mass spectrometry. Food Chem. 2014, 143, 199-204. [CrossRef]

30. Niu, Y.; Gao, B.; Slavin, M.; Zhang, X.; Yang, F.; Bao, J.; Yu, L. Phytochemical compositions, and antioxidant and anti-inflammatory properties of twenty-two red rice samples grown in Zhejiang. LWT-Food Sci. Technol. 2013, 54, 521-527. [CrossRef]

31. Zhang, X.; Gao, B.; Shi, H.; Slavin, M.; Huang, H.; Whent, M.; Yu, L.L. Chemical Composition of 13 Commercial Soybean Samples and Their Antioxidant and Anti-inflammatory Properties. J. Agric. Food Chem. 2012, 60, 10027-10034. [CrossRef]

32. Mazurek, B.; Chmiel, M.; Gorecka, B. Fatty Acids Analysis Using Gas Chromatography-Mass Spectrometer Detector (GC/MSD)— Method Validation Based on Berry Seed Extract Samples. Food Anal. Methods 2017, 10, 2868-2880. [CrossRef] 